\title{
ROLE OF ULTRASOUND IN THE EVALUATION OF BLUNT ABDOMINAL TRAUMA
}

\author{
Afshan Pathan
}

\begin{abstract}
This article provides an overview of the recent literature and research work about the growing role of ultrasound in assisting Emergency Physicians, Radiologists and Trauma Surgeons to make timely decision for the unstable patients with blunt abdominal trauma. Ultrasonography (US) in the evaluation of blunt trauma is now an accepted practice in the emergency departments. The main focus of the examination is detection of free fluid in the abdomen secondary to injury of the abdominal organs. The trauma patients in unstable condition and in whom significant free fluid is detected on US are immediately taken to the operating room for surgical exploration without undergoing computed tomographic (CT) correlation. US is also used to identify the hematoma surrounding the injured organ. The solid organ injuries such as the liver, spleen and kidney are also detected. However, US has limited value in the contained parenchymal injuries and injuries to the diaphragm, pancreas, adrenal gland and some bowel injuries. Thus, negative findings at US do not exclude an intraperitoneal injury, and close clinical observation or CT is warranted.
\end{abstract}

KEY WORDS: Abdomen. Trauma. Injury. Sonography.

\section{INTRODUCTION}

The care of a trauma patient is demanding and requires speed and efficiency. Evaluating patients who have sustained blunt abdominal trauma (BAT) remain one of the most challenging and resourceintensive aspects of acute trauma care. It is the need of emergency department that an optimal screening procedure for these patients should be less expensive, fast, accurate, easy to perform and portable. Ultrasonography (US) meets all these measures. Including this, Ultrasound can also be performed on pregnant patients, on patients with clotting disorders, and on patients with prior laparotomies and above all during trauma resuscitation without interfering with the therapeutic measures. An initial prospective investigation has demonstrated screening US to have a specificity of $96 \%$ and an overall accuracy of $96 \%$ in the detection of intraabdominal injury ${ }^{1}$.

Although ultrasound has been used for the investigation of urgent diagnostic dilemmas for almost 45 years, but during the past two decades ultrasound has achieved a primary role in the investigation of emergent conditions, notably in the trauma setting ${ }^{2}$. The use of US in evaluating blunt abdominal trauma was first reported in 1971 in Germany where Kristensen et $\mathrm{al}^{3}$ described its use in the diagnosis of splenic hematomas. Since late 1980s and early 1990s, US is used in several trauma centers in
Europe and Japan, but it was not until early 1990s that emergency physicians in the North America began showing interest in the use of US for blunt abdominal trauma ${ }^{4-6}$. The other modalities for the evaluation of BAT patient include diagnostic peritoneal lavage (DPL) and computed tomography (CT) of the abdomen. The advantages and disadvantages of these techniques are briefly reviewed in this article. Herein, we also review the typical US findings in hemoperitoneum and parenchymal injury. In addition, Free Fluid Scoring Systems, Focused Abdominal Sonography for Trauma (FAST), growing need for Serial Sonography and the limitations of US in the trauma setting are discussed in this article.

DIAGNOSTIC PERITONEAL LAVAGE: It has been used as a surgical tool for the diagnosis of hemoperitoneum since $1965^{7}$. It has shown sensitivity for intraperitoneal hemorrhage, as great as $95 \%{ }^{8}$, almost equal to US. But, it is insensitive to abdominal injuries that do not produce intraperitoneal hemorrhage. As a result, it cannot help detect some injuries of the retroperitoneum, pancreas and contained injuries to solid intraperitoneal organs. A number of studies ${ }^{9,10}$ advocate that US should replace DPL for the reasons; it is less costly, noninvasive, easily repeated, and is a bedside examination. In addition, US may provide the information about retroperitoneal hemorrhage, extent 
of hemoperitoneum, presence of parenchymal injury, pericardial effusion and haemothorax.

\section{ASSESMENT OF BAT PATIENTS WITH} COMPUTED TOMOGRAPHY: CT scan of the abdomen has been established as a sensitive mean of identifying intraperitoneal blood, detecting retroperitoneal hematomas and characterizing the magnitude of solid organ injuries even without hemoperitoneum. It is also more sensitive than other modalities for diaphragmatic, hollow viscus and retroperitoneal injuries ${ }^{11-13}$. In a prospective study carried out on hemodynamically stable patients who underwent both abdominal US and CT, it was concluded that US is highly sensitive for the detection of free intraperitoneal fluid but not sensitive for the identification of organ injuries. In hemodynamically stable patients, the value of US is mainly limited by the large percentage of organ injuries that are not associated with free fluid ${ }^{14}$. But, in comparison to US, the disadvantages of CT include higher costs, use of iodinated contrast medium that places the patient at risk of aspiration, and minimal radiation exposure. Another disadvantage of $\mathrm{CT}$ is that the patient needs transport, which may be problematic for the severely injured patient in unstable condition. Also, before CT, some adult and many pediatric patients may need sedation, which may increase the risk of airway compromise $^{15}$.

\section{HEMOPERITONEUM}

In evaluation of the abdominal cavity in BAT patients, the main focus is detection of free fluid. An abbreviated US examination for trauma proposed by Jehle et $\mathrm{al}^{16}$ reports sensitivity of $81.8 \%$ and specificity of $93.9 \%$ in the identification of hemoperitoneum in BAT patients. To some degree, hemoperitoneum always accompanies intraabdominal injury, with the exception of an intact subcapsular process. McKenney et $\mathrm{al}^{9}$ have reported solid organ injury without hemoperitoneum in $7 \%$ of BAT patients. Kimberley et $\mathrm{al}^{17}$ and Rozycki et $\mathrm{al}^{18}$ advocate the most common site of fluid accumulation, regardless of the site of injury as RUQ or more precisely the Morrison's pouch. Hahn et $\mathrm{al}^{19}$ retrospectively reviewed 539 patients with blunt trauma undergoing abdominal CT or abdominal US and intraperitonial fluid was identified in the following locations: Morrison's pouch (66\%), left upper quadrant $(56 \%)$, pelvis (48\%) and paracolic gutters $(36 \%)$. On the other hand, Levine et $\mathrm{al}^{20}$ retrospectively analyzed the CT scans of 60 patients with BAT and found that intraperitoneal fluid tended to accumulate in the pouch of Douglas (67\%) and Morrison's pouch (33\%). Free fluid will usually appear echo free but may be hypoechoic with a few internal echoes. At the site of injured solid organ, there is often echogenic blood (Figure I) that may be less obvious than the hypoechoic or echo free fluid so should not be overlooked ${ }^{15}$.

The minimum amount for detecting hemoperitoneum is a subject of interest. Kawaguchi and colleagues ${ }^{21}$ found that $70 \mathrm{ml}$ of blood could be detected, while Tiling et $\mathrm{al}^{22}$ found that $30 \mathrm{ml}$ is enough to be detected with ultrasound. They also concluded that a small anechoic stripe in the Morrison's pouch represents approximately $250 \mathrm{ml}$ of fluid, while $0.5 \mathrm{~cm}$ and $1 \mathrm{~cm}$ stripes represent approximately $500 \mathrm{ml}$ and $1 \mathrm{~L}$ of free fluid respectively.

Figure I: Severe splenic laceration in a 43-yearold man who had been assaulted. Longitudinal US scan of the left upper quadrant of abdomen shows that spleen is surrounded by a slightly hyperechoic rim (arrow). Free fluid was present in the abdomen. The patient was taken to the

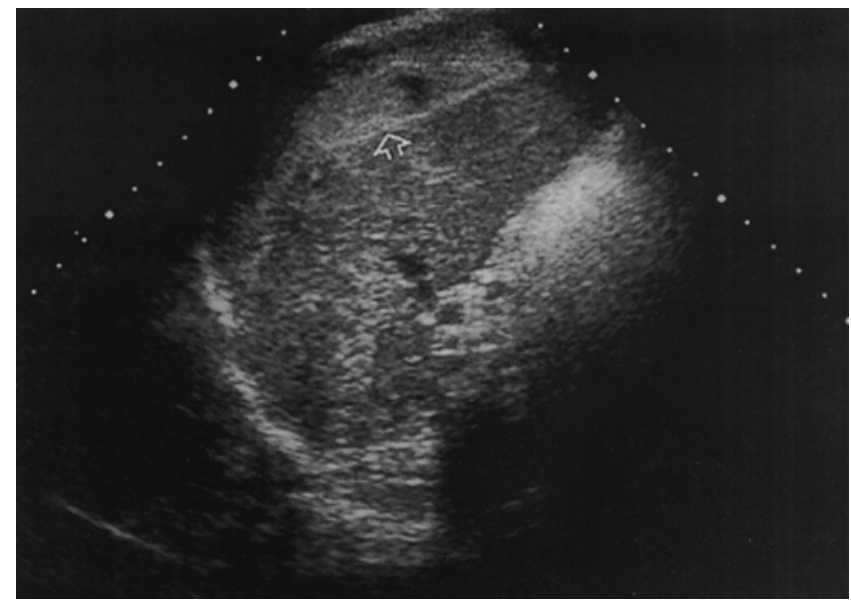

operating room for splenectomy.

(Reprinted with permission from reference 15)

\section{FREE FLUID SCORING SYSTEMS}

It is now well accepted that hemoperitoneum following trauma is not necessarily an indication for immediate laparotomy. Although, US can demonstrate the extent of hemoperitoneum, but this information to the surgeon has been limited to the use of words such as "mild," "moderate," or "massive" to describe fluid volume. To improve this insufficient information and assist the surgeon in decision making, in 1994, a scoring system for fluid quantification was developed at the Department of Radiology, Duke University Medical Center ${ }^{23}$. On US, the depth of the largest fluid collection is measured from anterior to posterior in centimeters and each additional site where fluid is present is given one point. The patient's hemoperitoneum score is calculated by adding the depth of the largest collection and the total number of points assigned to 
all the additional sites that demonstrate fluid (In small, curvilinear collections; the width of fluid is determined). For example, if longitudinal US image of the pelvis reveals the largest collection of fluid, whose depth $(9 \mathrm{~cm})$ was determined by measuring the fluid from anterior to posterior. And longitudinal US image of the Morrison's pouch also demonstrates fluid. One point was added for this site, resulting in a hemoperitoneum score of $10(9+1)$. A retrospective study has showed that $90 \%$ of patients with a score of 2 or less were managed conservatively whereas $75 \%$ of patients with a score of higher than 2 required laparotomy. These results suggest that quantifying free fluid during the early stages of assessment may improve patient selection for laparotomy. So, at that institution on surgeon's request, a hemoperitoneum score is now included for all patients with intraperitoneal hemorrhage seen on US images.

Huang et $\mathrm{al}^{24}$ has developed another scoring system. They based criteria on locating pockets of fluid with a thickness of $2 \mathrm{~mm}$ or greater. Each region or pocket of fluid $2 \mathrm{~mm}$ or greater received a score of 1 . Patients with 3 pockets, or a score of 3 or greater were taken to the operating room. Other scoring systems $^{21,25}$ are also developed at different trauma centers to improve the patient selection for laparotomy in patients with BAT.

\section{PARENCHYMAL INJURIES}

CT has remained the standard of reference for evaluating organ parenchyma in patients with blunt trauma ${ }^{26}$. However, valuable information regarding the presence of parenchymal injury may be obtained at US evaluation of the acutely injured patient. Rothlin et $\mathrm{al}^{27}$ have reported the sensitivity of $41.4 \%$ to detect parenchymal injuries on US. Hematoma and localized lacerations manifest as regions of increased echogenicity that over time with the onset of hemolysis will become anechoic. The extensive parenchymal injury manifests in the liver as widespread architectural disruption with absence of the normal vascular pattern, whereas extensive splenic injury frequently manifests as a diffusely heterogeneous parenchymal pattern with both hyper and hypoechoic regions ${ }^{28}$.

Spleen: It is the most commonly injured organ in blunt abdominal trauma. Richards and colleagues ${ }^{29}$ identified parenchymal injuries of the spleen in 31 of 162 BAT patients. The most common pattern of laceration was a diffuse heterogeneous appearance, seen in 14 cases. Discrete hyperechoic or hypoechoic regions within the traumatized spleen may also be identified with US. A hyperechoic or hypoechoic perisplenic rim or crescent, representing a clot often surrounds the spleen. Blunt splenic trauma can also result in subcapsular and intraparenchymal hematoma. In case of splenic rupture splenic enlargement, a diffusely heterogeneous parenchymal pattern and change in the contour of the splenic border is seen.

Liver: The liver is the third most common organ injured in the abdomen after the spleen and kidney. Richards et $\mathrm{al}^{30}$ has observed that sonography may also reveal blunt hepatic injury with three distinct patterns. The most common US pattern observed in 10 patients was a discrete hyperechoic area. A diffuse hyperechoic pattern was seen in six cases, and a discrete hypoechoic pattern in two cases. An echogenic clot often surrounds the liver, and hypoechoic fluid may be in other portions of the abdomen. Hepatic lacerations appear more hypoechoic or cystic when they are scanned days after the initial injury (Figure II $\mathbf{a}, \mathbf{b}, \mathbf{c})^{15}$.

Kidney: Miller et $\mathrm{al}^{31}$ have reported that $90 \%$ of renal injuries result from blunt trauma. Although, renal lacerations and hematomas can be identified and delineated on ultrasonogram, but it is more likely to be abnormal with severe (grade II or greater) renal injuries. The limitations of ultrasound include inability to distinguish fresh blood from extravasated urine and inability to identify vascular pedicle injuries or segmental infarcts. With close color and pulsed Doppler interrogation, a vascular injury can be diagnosed. But, CT remains the best screening method to overcome the above mentioned limitation of US for BAT patients with renal injuries ${ }^{32-34}$. However, ultrasound has a place in the follow up of these patients e.g. to asses the progress or resolution of hematoma.

\section{FOCUSED ASSESSMENT WITH SONOGRAPHY FOR TRAUMA}

Although, the use of sonography in the detection of abdominal parenchymal injuries and hemoperitoneum is not new but it has only been in the 1990s that sonography is more widely advocated for the screening evaluation of patients with $\mathrm{BAT}^{35}$. Focused abdominal ultrasonography has been introduced in Europe for years but has only recently been introduced in the United States. The first American report of physician-performed abdominal ultrasound in the evaluation of BAT was published in 1992 by Tso and colleagues ${ }^{36}$. Since then, numerous articles have been published in the United States supporting the use of ultrasound in the evaluation of patient with BAT. Most recently, the FAST has been included as part of the advanced trauma life support course.

The FAST examination has virtually replaced DPL as 
the procedure of choice in the evaluation of hemodynamically unstable trauma patients. The sensitivity of FAST scan is $98 \%$ and specificity $95 \%{ }^{37}$. Figure II: Liver laceration in a 33-year-old man involved in a motor vehicle accident.

(a)

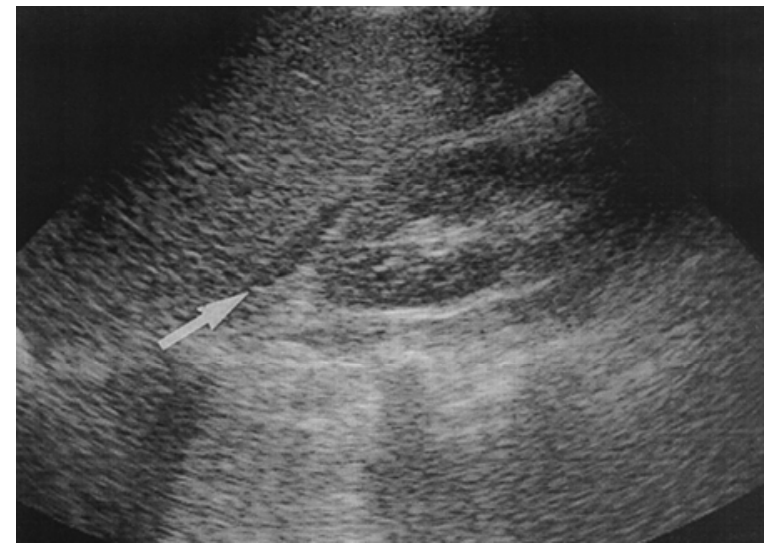

Longitudinal US scan of right upper quadrant of abdomen shows a small amount of free fluid in

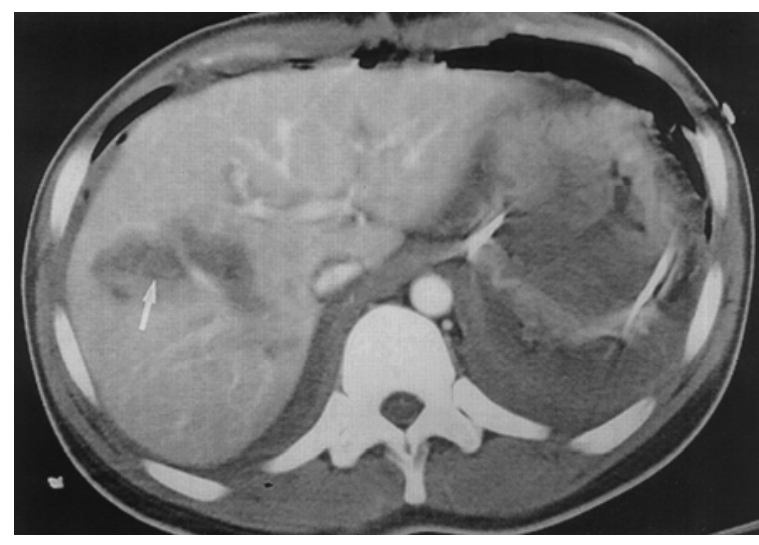

renal fossa (arrow) and fairly normal appearance of the liver.

(b)

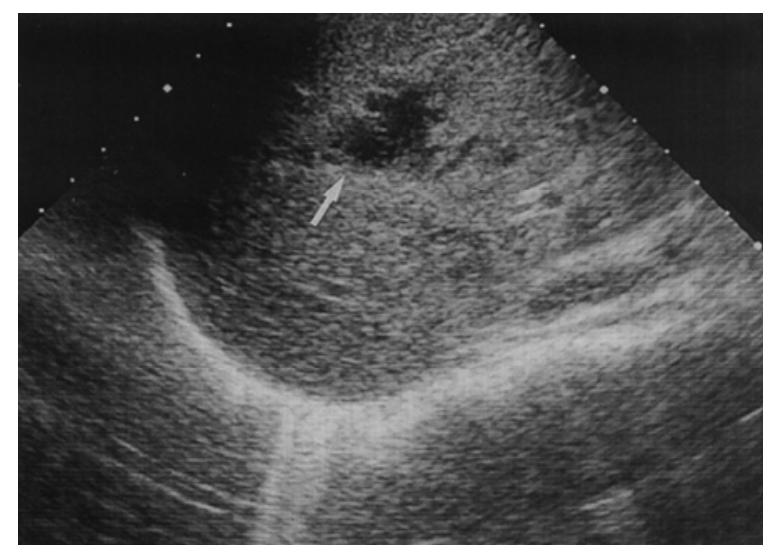

CT scan obtained at the same time as the US scan in (a) shows among other findings, a large liver laceration (arrow).

(c) Follow-up US scan obtained 13 days later shows a hypoechoic region (arrow) in the right lobe of the liver that represents patient's resolving liver laceration.

(Reprinted with permission from reference 15)

FAST is performed by trauma surgeons, emergency physicians or radiologists in the Emergency Department. The examination takes only a few minutes to perform. McGahan et $\mathrm{al}^{38}$ have defined that most crucial portion of focused abdominal US for trauma is evaluation of the right upper quadrant, left upper quadrant and pelvis for free fluid. Many patients in trauma setting have an empty bladder with placement of a Foley's catheter. Thus, free fluid in the pelvis may be overlooked. If detection of free fluid in the pelvis is desired, the patient should not have an empty bladder ${ }^{38-40}$. FAST also examines the right and left paracolic gutters, epigastrium, the pleural and pericardial spaces ${ }^{38}$. More recently, there have been some studies to show that it may be of use in detecting pneumothorax ${ }^{41,42}$.

\section{SERIAL SONOGRAPHY}

The value of serial sonography has not been fully investigated in patients with BAT. Studies suggest that serial sonography should be performed as a part of the follow-up physical examination ${ }^{43,44}$. Because in many cases admitted to trauma center, the time from injury to diagnosis is not more than 1 hour. This brief time may not always be sufficient to manifest the hemoperitoneum. Henderson et $\mathrm{al}^{43}$ identified four patients whose initial US results were negative for hemoperitoneum and later became positive at serial examinations. Furthermore, the isoechoic lacerations may be missed in the first few hours of trauma. Over the time, with the onset of hemolysis, they become hypoechoic or cystic and can be detected when scanned days after the initial injury (Figure II $\mathbf{a}, \mathbf{b}, \mathbf{c})^{15}$. In many institutions, it is advocated that to avoid 
missing significant injuries, all patients with blunt abdominal trauma having negative findings on initial US should be observed for several hours after imaging and serial ultrasound examinations should be made every 1 to 2 hours for the first 6 hours after admission, and then every 12 hours for the next 2 days $^{17}$.

\section{LIMITATIONS OF ULTRASONOGRAPHY}

Limitations of US include its dependence on operator's skill, which becomes particularly important if surgeons or emergency physicians have limited training. Adequate training and experience are crucial for accurate US evaluation ${ }^{1}$.

In the obese patient, use of a 3.5-MHZ transducer may be adequate for the exclusion of intraperitoneal fluid but does not usually permit adequate assessment of the organ parenchyma. In the clinically stable patient, CT is the preferred modality for complete evaluation ${ }^{15}$. Rarely, the presence of subcutaneous emphysema may prevent adequate US examination. Subcutaneous air from a pneumothorax that dissects inferiorly may collect over the liver or spleen and prevent adequate imaging. The left upper quadrant, where the spleen provides only a small acoustic window can easily be obscured by air and that area is not completely imaged; in such cases, CT or diagnostic peritoneal lavage should be performed $^{17}$.

There is a number of articles that have pointed out that if US alone is used to evaluate blunt trauma victims, contained parenchymal injuries which may not be accompanied by hemoperitoneum and some bowel and mesenteric injuries may go undetected $^{1,9,39,40,45}$. Finally, there is little doubt that sonography will be limited or unable to show certain types of injuries. These are not restricted to but include spinal and pelvic fractures, diaphragmatic ruptures, vascular injuries, pancreatic injuries and adrenal injuries ${ }^{1,40}$.

\section{CONCLUSION}

The role of ultrasound in the assessment of blunt abdominal trauma continued to grow in the past decade. In many trauma centers, US have become the screening modality of choice in blunt abdominal trauma as it can help determine the need for surgical intervention within minutes of a patient's arrival. In many institutions, US has replaced diagnostic peritoneal lavage and has redefined the role of CT in the immediate evaluation of the trauma patient which may be performed if urgent laparotomy is not required. Serial examinations appear to increase further the sensitivity of ultrasound. Those using sonography in this group of patients should be aware of its uses but also its pitfalls and limitations. At the same time, they need a close interaction with the surgical team to reduce the risk of missed injury.

\section{REFERENCES}

1. Brown MA, Casola G, Sirlin CB et al. Blunt Abdominal Trauma: Screening US in 2,693 Patients. Radiology 2001; 218: 352-58.

2. Rozycki GS, Newman PG. Surgeon-performed ultrasound for the assessment of abdominal injuries. Adv Surg 1999; 33: 243-59.

3. Kristensen JK, Buemann B, Kuhl E. Ultrasonic scanning in the diagnosis of splenic haematomas. Acta Chir Scand 1971; 137: 65357.

4. Jehle D, Guarino J, Karamanoukian $H$. Emergency department ultrasound in the evaluation of blunt abdominal trauma. Am J Emerg Med 1993; 11: 342-46.

5. Richards JR, Knopf NA, Wang $L$ et al. Blunt abdominal trauma in children: evaluation with emergency US. Radiology 2002; 222: 749-54.

6. Ballard RB, Rozycki GS, Knudson MM et al. The surgeon's use of ultrasound in the acute setting. Surg Clin North Am 1998; 78(2): 337-64.

7. Root HD, Hauser CW, McKinley CR et al. Diagnostic peritoneal lavage. Surgery 1965; 57: 633-37.

8. Day AC, Rankin N, Charlesworth P. Diagnostic peritoneal lavage: integration with clinical information to improve diagnostic performance. $\mathrm{J}$ Trauma 1992; 32: 52-57.

9. McKenney M, Lentz K, Nunez D et al. Can ultrasound replace diagnostic peritoneal lavage in the assessment of blunt trauma?. J Trauma 1994; 37: 439-41.

10. Liu M, Lee CH, P'eng FK. Prospective comparison of diagnostic peritoneal lavage, computed tomographic scanning and ultrasonography for the diagnosis of blunt abdominal trauma. J Trauma 1993; 35: 267-70.

11. Garber BG, Bigelow E, Yelle JD et al. Use of abdominal computed tomography in blunt trauma: do we scan too much? Can J Surg 2000; 43:1621.

12. Kinnunen J, Kivioja A, Poussa $K$ et al. Emergency CT in blunt abdominal trauma of multiple injury patients. Acta Radiol 1994; 35: 319-22.

13. Kshitish $M$, Sushma $V$, Sanjay $T$ et al. Comparative evaluation of ultrasonography and CT in patients with abdominal trauma: a prospective study Ind J Radiology 2000; 10: 4.

14. Poletti PA, Kinkel $K$ et al. Blunt abdominal trauma: should US be used to detect both free 
fluid and organ injuries? Radiology 2003;227:95103.

15. McGahan JP, Wang L, Richards JR. Focused abdominal US for trauma. Radiographics 2001; 21: S191-99.

16. Jehle D, Guarino J, Karamanoukian $H$. Emergency department ultrasound in the evaluation of blunt abdominal trauma. Am J Emerg Med 1993; 11:342-46.

17. McKenney KL. Role of US in the diagnosis of intraabdominal catastrophies. Radiographics 1999; 19:1332-39.

18. Rozycki GS, Ochsner MG, Feliciano DV et al. Early detection of hemoperitoneum by ultrasound examination of the right upper quadrant: a multicenter study. J Trauma 1998; 45(5): 878-83.

19. Hahn DD, Offerman SR, Holmes JF. Clinical importance of intraperitoneal fluid in patients with blunt intraabdominal injury. Am J Emerg Med 2002; 20(7): 595-600.

20. Levine CD, Patel UJ, Wachsberg RH. CT in patients with blunt abdominal trauma: clinical significance of intraperitoneal fluid detected on a scan with otherwise normal findings. Am J Roentgenol 1995;164(6):1381-5.

21. Kawaguchi S, Toyonaga J, Ikeda K. Five point method: an ultrasonographic quantification formula of intraabdominal fluid collection. Jpn J Acute Med 1987; 7: 993-7.

22. Tiling $\mathrm{T}$, Boulion $\mathrm{B}$, Schmid $\mathrm{A}$ et al. Ultrasound in blunt abdominothoracic trauma. In Border JR, ed. Blunt Multiple Trauma: Comprehensive Pathophysiology and Care. New York, NY: Marcel Dekker; 1990: 415-33.

23. McKenney KL, McKenney MG, Nunez DB et al. Interpreting the trauma ultrasound: observations in 62 positive cases. Emerg Radiol 1996; 3:11317.

24. Huang MS, Liu M, Wu JK et al. Ultrasonography for the evaluation of hemoperitoneum during resuscitation: a simple scoring system. J Trauma 1994; 36:173-77.

25. Sirlin CB, Casola G, Brown MA et al. Quantification of fluid on screening ultrasonography for blunt abdominal trauma: a simple scoring system to predict severity of injury. J Ultrasound Med 2001; 20(4): 359-64.

26. Brown MA, Casola G, Sirlin CB et al. Importance of evaluating organ parenchyma during screening abdominal ultrasonography after blunt trauma. $\mathrm{J}$ Ultrasound Med 2001; 20(6): 577-83; quiz 585.

27. Rothlin MA, Naf R, Amgwerd $M$ et al. Ultrasound in blunt abdominal and thoracic trauma. J Trauma 1993; 34:488-95.

28. von Sonnenberg E, Simeone JF, Mueller PR et al. Sonographic appearance of hematoma in liver, spleen and kidney: a clinical, pathologic and animal study. Radiology 1983; 147:507-10.

29. Richards JR, McGahan JP, Jones CD et al. Ultrasound detection of blunt splenic injury. Injury 2001; 32:95-103.

30. Richards JR, McGahan JP, Pali MJ et al. Sonographic detection of blunt hepatic trauma: hemoperitoneum and parenchymal patterns of injury. Trauma 1999; 47:1092-97.

31. Miller KS, McAninch JW. Radiographic assessment of renal trauma: our 15 years experience. J Urol 1995; 154:352.

32. McAninch JW, Federle MP. Evaluation of renal injuries with computed tomography. J Urol 1982; 128:456.

33. Perry MJ, Porte ME, Urwin GH. Limitations of ultrasound evaluation in acute closed renal trauma. J R Coll Surg Edinb 1997; 42:420-22.

34. McGahan JP, Richards JR, Jones CD et al. The use of ultrasound in acute renal trauma. Radiology 1998; 209(P):496.

35. Kimura A, Otsuka T. Emergency center ultrasonography in the evaluation of hemoperitoneum: a prospective study. J Trauma 1991; 31:20-23.

36. Tso P, Rodriquez A, Cooper $C$ et al. Sonography in blunt abdominal trauma: a preliminary progress report. J Trauma 1992; 33: 39-44.

37. Lingawi SS, Buckley AR. Focused abdominal US in patients with trauma. Radiology 2000; 217(2): 426-9.

38. McGahan JP, Richards J, Fogata ML. Emergency ultrasound in trauma patients. Radiol Clin North Am 2004;42(2):417-25.

\author{
AUTHOR AFFILIATION: \\ Dr. Afshan Pathan \\ Department of Diagnostic Radiology and Imaging \\ Liaquat University Hospital Hyderabad / Jamshoro, Sindh - Pakistan
}

